\title{
METODOLOGÍA PARA DETERMINAR ATRIBUTOS Y MÉTRICAS DE CALIDAD EN SISTEMAS HIPERMEDIA ADAPTATIVOS EDUCATIVOS BASADOS EN ESTILOS DE APRENDIZAJE
}

\author{
Helmut Leighton Álvarez $z^{1}$ \\ Marcela Prieto Ferraro ${ }^{1}$ \\ Francisco José García Peñalvo ${ }^{2}$ \\ 1 Universidad de Salamanca, Instituto Universitario de Ciencias de la Educación (IUCE), España, \\ \{hleighton,mprieto5\}@usuarios.retecal.es \\ 2 Universidad de Salamanca, Departamento de Informática y Automática, España, \\ fgarcia@usal.es
}

\begin{abstract}
Resumen: Este trabajo es una propuesta metodológica para determinar atributos y métricas de calidad en sistemas hipermedia adaptativos educativos basados en estilos de aprendizaje, específicamente en los estilos activo, reflexivo, teórico y pragmático. Estos atributos y métricas están referidos únicamente a la interacción del usuario-estudiante con el sistema, es decir, desde la óptica puramente educativa y no desde el punto de vista de la herramienta informática como tal.

Para ello comienza su análisis desde las características del estilo de aprendizaje y a partir de ellas se procede al establecimiento de los atributos de acuerdo a las estrategias instruccionales que le correspondan. Finalmente, se definen las métricas necesarias para cada uno de los atributos, estableciendo el o los tipos de variables que involucran, sus unidades de medida y escalas. De esta manera se desarrolla una metodología de cascada de determinación de atributos y métricas.
\end{abstract}

Palabras clave: Sistemas hipermedia adaptativos, sistemas hipermedia adaptativos basados en estilos de aprendizaje, atributos de calidad, métricas.

\section{Introducción}

Los sistemas hipermedia adaptativos se adaptan a las características propias del usuario en general y a las características específicas del usuario-estudiante cuando este sistema es parte del proceso educativo, respondiendo a las limitaciones presentadas en los sistemas hipermedia que los precedieron. Estos nuevos sistemas pretenden evitar la desorientación de los estudiantes al navegar por el sitio, disminuir la fatiga cognitiva y no tratar a cada usuario-estudiante bajo un mismo enfoque o estrategia instruccional (Prieto, Gros, García, 2003). En nuestro caso consideramos la adaptación basada en los estilos de aprendizaje, y específicamente en la taxonomía propuesta por Alonso, Gallego, Honey (1997).

Sin embargo, surge la necesidad de establecer una metodología de evaluación de calidad de estos sistemas. Existen algunas metodologías de evaluación de calidad para aplicaciones software y para aplicaciones $W e b$, pero están referidas solamente a las aplicaciones como tal y a su usabilidad. En este sentido, es preciso ampliar estas 


\begin{abstract}
This study is a methodological proposal for determining the attributes and metrics of quality in adaptative educational hypermedia systems based on learning styles, specifically,concerning the active, reflexive, theoretical and pragmatic styles. These attributes and metrics refer only to the interaction of the user-student with the system, i.e., the study is done from a purely educational perspective, not from the perspective of the computer as a tool. Thus, the analysis takes as a starting point the characteristic o the learning style. Based on this, he attributes are established in accordance with the corresponding instructional strategies. Finally, the metrics needed for each of the attributes are defined, and the types of variables involved, their units of measurement and sales are established. In this way a cascade methodology is developed for determining attributes and metrics.
\end{abstract}

Key words: Adaptive hypermedia systems, adaptive hypermedia systems based on learning styles, quality attributes, metrics. metodologías o crear nuevos procedimientos de calidad y estandarización desde un enfoque propiamente educativo (Prieto, Leighton y García 2004; Leighton y García, 2004a; Leighton, García y López, 2004b).

Así, el objetivo de este trabajo es proponer una metodología de evaluación de calidad de los Sistemas Hipermedia Adaptativos Educativos (SHAE) basados en estilos de aprendizaje. Para ello, la metodología propuesta comienza con un cruzamiento entre cada estilo de la taxonomía mencionada y las estrategias instruccionales planteadas por Reigeluth y Moore (1999), de esta forma, se establecen los atributos de calidad, las métricas correspondientes a cada uno de ellos y su definición.

Para este objetivo planteado, en este trabajo se presenta en el punto 2 los atributos de calidad ampliamente discutidos en la literatura, la teoría sobre métricas de calidad y su definición en el punto 3 , la metodología propuesta en el punto 4, para finalmente concluir en el punto 5 .

\section{Atributos de calidad}

Existe una serie de atributos observables directa o indirectamente que determinan la calidad Web, dando la medida de estos atributos un valor de estimación de la calidad total del sitio. En la literatura y propuestas recientes (Bevan, Kirakowski, y Maissel, 1991; Bevan, 1999; Leighton y García, 2003; Nielsen, 1993; Olsina, 1999, 2002; Olsina, Bertoa, Lafuente, Martín, Katrib, y Vallecillo, 2002; Olsina, Lafuente, y Rossi, 2001) se plantean como atributos de calidad la usabilidad, funcionalidad, fiabilidad, eficiencia, mantenibilidad y operabilidad.

Cada uno de estos atributos corresponden a puntos de vista opuestos cuando se estudia un sitio Web, esto es, desde la perspectiva de los desarrolladores del sitio y su funcionamiento y, por otro lado, desde la perspectiva del usuario y de cómo él ve la funcionalidad global. Estos atributos 
se pueden perfectamente extrapolar a los SHA en Web.

El modelo de calidad Web denominado WebSite-QEM, planteado por (Olsina, 2002, 1999; Olsina et al., 2002), es una propuesta para llevar a cabo esta medición de calidad y prueban un modelo basado en la inspección, con sistemas manuales y automáticos de cada uno de los cinco aspectos que considera para ella, usabilidad, funcionalidad, fiabilidad, eficiencia y capacidad de mantenimiento. La motivación que se presenta en nuestra propuesta radica, a diferencia de WebSite-QEM, en que los atributos a considerar en la calidad de los SHAEs están dirigidos exclusivamente a aspectos pedagógicos de interacción y de tipo de actividad a desarrollar por los usuarios-estudiantes.

\section{Métricas de calidad}

Un marco conceptual para la definición y explotación de métricas se plantean en (Olsina et al., 2002) donde se definen para ello los conceptos entidad y atributos, la entidad representa un objeto, tangible o intangible, que exhibe un comportamiento observable en el mundo real como por ejemplo, un proceso. Cómo estas entidades no pueden medirse directamente, la medición se lleva a efecto por medio de los atributos que se le pretenden. Las entidades pueden dividirse en sub-entidades, cada una de las cuales tendrá sus propios atributos o propiedades, y desde el punto de vista de la medición a todas las entidades se les puede atribuir atributos.

Un atributo puede ser cuantificado por varias métricas, dependiendo de cómo se realice la medición, esto es, un texto puede ser medido por la cantidad de caracteres, palabras o tamaño en bytes.

Lo anterior significa que una métrica debe ser comprendida en relación a los atributos que está cuantificando y a la entidad o sub-entidad que se encuentra asociada. Esto implica, además, identificar claramente el tipo de valor que se obtiene, en qué unidad se expresa ese valor y el tipo de escala que se usa, para poder realizar posteriormente un correcto análisis matemático y de interpretación.

El protocolo corresponde a la forma de realizar la recolección de datos, las reglas de procedimiento y conteo, de esta manera es posible analizar y derivar, en algunos casos, a herramientas automáticas o semi-automáticas que realicen esta tarea, en este caso el protocolo corresponde al algoritmo utilizado para recolectar los datos, dejando claramente establecido que una métrica puede tener uno o más protocolos. El fin último del protocolo es asegurar la repetitividad y replicabilidad del proceso de medición.

Las métricas pueden ser directas o indirectas, y están en estrecha relación con la cuantificación directa o indirecta de los atributos a los cuales se encuentran asociadas. En el caso de las métricas indirectas es necesario determinar cuál es la ecuación que formaliza ese atributo indirecto. Los atributos directos no se pueden descomponer, y los atributos indirectos están conformados por una relación de atributos directos.

De esta forma, este modelo permite representar a los atributos relacionados con sus entidades y sub-entidades como a la jerarquía de las entidades y sub-entidades, dando así una visión "navegable" desde el punto de vista de las entidades y atributos del sitio Web a evaluar.

\section{Propuesta metodológica}

La metodología que se propone para medir la calidad en un SHAE basado en estilos de aprendizaje tiene dos puntos fundamentales de estudio, la interacción del sistema con el usuario y el tipo de actividad que este sistema propone al usuario, considerando en este artículo lo referido a la interacción.

Para que un SHAE basado en Estilos de Aprendizaje sea considerado de calidad 
en el aspecto pedagógico, éste debe cumplir con la interacción sistema-usuario y con las actividades requeridas de acuerdo al estilo adaptado, es decir, ya no sólo hablamos de contenidos, de la presentación de los mismos, de los medios disponibles y de las posibilidades de navegación, aquí valoramos las estrategias pedagógicas que adopta el sistema de acuerdo a las características del usuario. Esto significa que debemos determinar la forma en que definiremos los atributos del sistema y sus respectivas métricas (Prieto et al., 2004).

\subsection{Determinación de atributos y métricas}

Para determinar los atributos de calidad y sus métricas, como muestra la figura 1 , se deben considerar las características del estilo, cuáles son esas especificaciones que hacen diferente al usuario-estudiante de otros (Leighton et al., 2004a; Leighton et al., 2004b).

Una vez clarificadas y definidas estas especificaciones se procede a definir los atributos de acuerdo a las estrategias

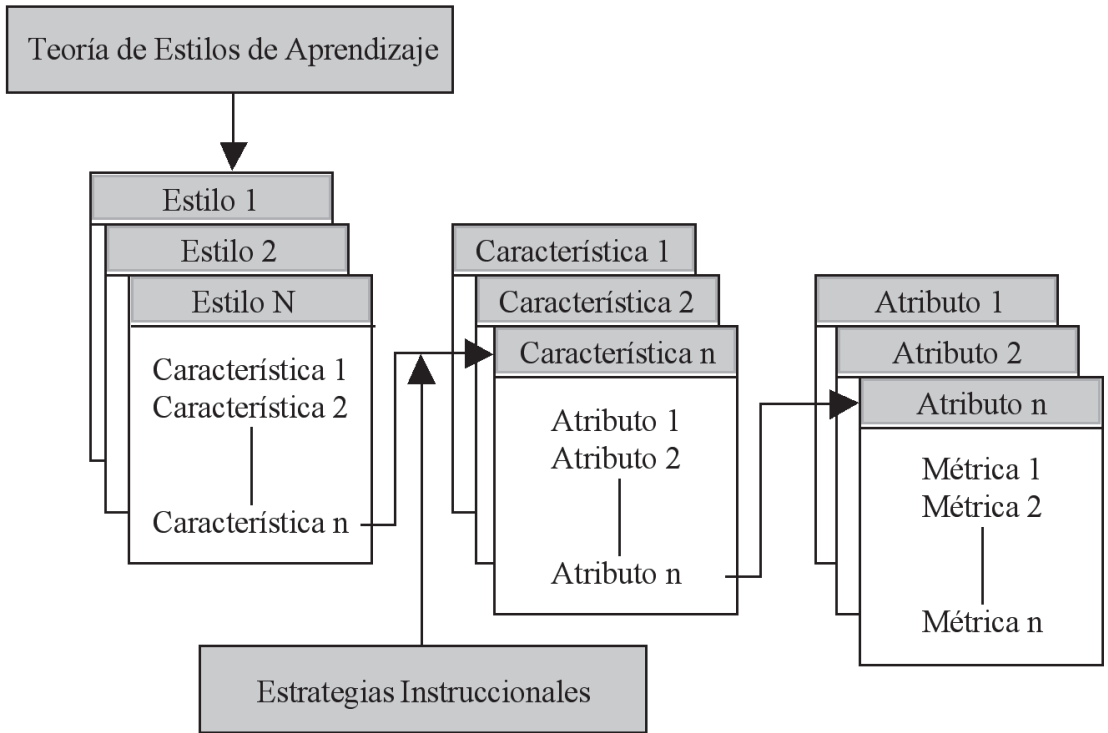

Fig. 1. Determinación de métricas y atributos en SHAEs basados en Estilos de Aprendizaje.

instruccionales que determinan la interacción sistema-estudiante.

Determinados los atributos se procede a definir las métricas necesarias para cada uno de ellos, de tal forma que permitan medir la presencia y en qué grado se encuentran presentes en el sistema.

Como ejemplo tomamos un SHAE basado en los estilos de aprendizaje de Alonso et al., (1997), en la tabla 1 se han cruzado las características establecidas para los estilos y las estrategias instruccionales propuestas por Reigeluth y Moore (1999).

Una vez establecidas las estrategias que debieran desarrollarse en concordancia con el estilo del usuario, comenzamos a determinar las métricas que nos permitirán medir el grado de presencia de los atributos (Leighton et al., 2004a; Leighton et al., 2004b). De esta forma, como vemos en la tabla 2, se establecen una o más métricas para cada atributo.

\subsection{Definición formal de métricas}

Para definir las métricas es necesario establecer el tipo de criterio elemental que las rige, que en el caso de las propuestas en este trabajo corresponden a binario con variable discreta, multinivel con variable 
discreta y multivariable discreta, y que en este trabajo solo detallaremos las correspondientes al estilo activo de aprendizaje.

El criterio elemental binario con variable discreta corresponde a la presencia o no presencia de la variable en estudio, variable directa, como en los casos de las métricas 1.1.1, 1.1.2 1.1.3, 1.1.4, 1.2.1, 1.4.1 y 1.4.2, ver tabla 2, quedando el criterio elemental como sigue: $\mathrm{CrE}(\mathrm{Xi})=\{(0,0)$, $(1,100)\}$, donde 0 significa ausencia $0 \%$ y 1 significa presencia $100 \%$, y la escala de preferencia definida como:

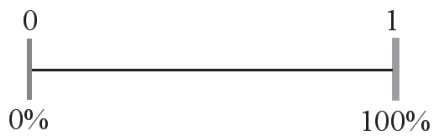

Tabla 1

Características de los estilos activo, reflexivo, teórico y pragmático v/s estrategias instruccionales

\begin{tabular}{lll}
\hline Estilo & \multicolumn{1}{c}{ Características } & \multicolumn{1}{c}{ Estrategias instruccionales } \\
& - & Intentar cosas nuevas, nuevas experiencias, nuevas \\
& oportunidades. & Control centrado en el estu- \\
& - & diante. \\
Activo & personas de mentalidad semejante para dialogar, dirigir deba- - & Trabajo en pares, equipos \\
& tes y reuniones. & y/o grupos. \\
& - & Generar ideas sin formalismos ni estructura, cambiar y variar - \\
& las cosas, arriesgarse, sentirse ante un reto con recursos & \\
& inadecuados, intentar algo diferente.
\end{tabular}

- Observar, investigar detenidamente, reunir información, escu- - Control más centrado en el char, tener posibilidades de leer o prepararse de antemano, profesor. escuchar puntos de vistas diferentes, intercambiar opiniones - Trabajo orientado al domicon otros previo acuerdo. nio y tópico.

Reflexivo - Trabajar sin presiones ni plazos, pensar antes de actuar, asi- - Trabajo individual más que milar antes de comentar. grupal.

- Hacer análisis detallados, realizar informes cuidadosamente - Soporte cognitivo. ponderados, trabajar concienzudamente.

- Sentirse en situaciones estructuradas con finalidad clara, - Control centrado en el explorar metódicamente las asociaciones y las relaciones entre profesor. ideas, acontecimientos y situaciones, recabar todos los datos - Trabajo orientado al tópico de un sistema, modelo, concepto o teoría. y dominio.

- Participar en sesiones de pregunta y respuesta, posibilidad de - Trabajo individual y grupal Teórico cuestionar, leer o escuchar sobre ideas y conceptos, encontrar - Soporte cognitivo. ideas y conceptos complejos.

- Sentirse intelectualmente presionado, poner a prueba métodos y lógica, participar en situaciones complejas, llegar a entender acontecimientos complejos.

- Aprender técnicas para hacer las cosas con ventajas prácticas, - Control centrado en el tener un modelo a seguir, adquirir técnicas aplicables de inme- profesor. diato, aplicación inmediata de lo aprendido, recibir muchas - Trabajo orientado al indicaciones prácticas y técnicas. dominio y problema.

Pragmático - Resolver problemas reales, uso de simulaciones, poder practi- - Trabajo en pares, equipos car y experimentar técnicas con asesoramiento e información, y/o grupos. relación de tema tratado con problemas.

Soporte cognitivo.

- Tratar con expertos que saben hacer las cosas ellos mismos. 
La métrica 1.1.1 de la tabla 2 ejemplifica este tipo de criterio en la tabla 3

El criterio elemental multinivel con variable discreta corresponde a la no presencia o a la presencia en dos o más niveles de la variable en estudio, variable directa, como en los casos 1.2.2, 1.3.1 y 1.3.2 de la tabla 2, quedando el criterio elemental definido como $\mathrm{CrE}(\mathrm{Xi})=\{(0,0),(1,60),(2,100)\}$, donde 0 significa ausencia 0\%, 1 significa presencia básica $60 \%$ y 3 presencia básica más un plus $100 \%$, en el caso de tres niveles, y su escala de preferencia definida como:

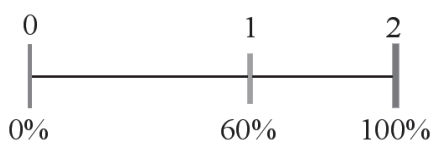

Tabla 2

Atributos y métricas para los estilos activo, reflexivo, teórico y pragmático

\begin{tabular}{ccc}
\hline $\begin{array}{c}\text { Estilo de } \\
\text { aprendizaje }\end{array}$ & Atributos & Métricas \\
\hline
\end{tabular}

1.1 Control centrado en el estudiante

1.2 Trabajo interdisciplinario

Activo

1.3 Trabajo en pares, equipos y/o grupos

1.4 Interacción con pares

2.1 Control más centrado en el profesor

2.2 Trabajo orientado al dominio y tópico

Reflexivo

2.3 Trabajo individual más que grupal

2.4 Soporte cognitivo
1.1.1 Grado de determinación de objetivos

1.1.2 Grado de selección de contenidos

1.1.3 Grado de opciones de navegación

1.1.4 Grado de selección de actividades

1.1.5 Grado de decisión

1.2.1 Número de materias

1.2.2 Número de áreas

1.3.1 Relación actividades individuales v/s actividades grupales

1.3.2 Número de miembros por actividad

1.4.1 Intercambio vía foros

1.4.2 Intercambio vía correo electrónico

2.1.1 Grado de determinación de objetivos

2.1.2 Grado de selección de contenidos

2.1.3 Grado de opciones de navegación

2.1.4 Grado de selección de actividades

2.1.5 Grado de decisión

2.2.1 Grado de orientación al dominio

2.2.2 Grado de orientación al tópico

2.2.3 Grado de orientación al trabajo

2.3.1 Relación de actividades individuales $\mathrm{v} / \mathrm{s}$ grupales

2.4.1 Interacción con material impreso

2.4.2 Interacción con material audiovisual

2.4.3 Intercambio con pares

2.4.4 Intercambio con expertos 
Estilo de aprendizaje

Atributos

3.1 Control centrado en el profesor

3.2 Trabajo orientado al tópico y dominio

Teórico

3.3 Trabajo individual y grupal

3.4 Soporte cognitivo
3.1.1 Grado de determinación de objetivos

3.1.2 Grado de selección de contenidos

3.1.3 Grado de opciones de navegación

3.1.4 Grado de selección de actividades

3.1.5 Grado de decisión

3.2.1 Grado de orientación al dominio

3.2.2 Grado de orientación al tópico

3.2.3 Grado de orientación al trabajo

3.3.1 Trabajo individual

3.3.2 Trabajo en equipo

3.3.3 Trabajo grupal

3.3.4 Grado de agrupación

3.4.1 Interacción con expertos

3.4.2 Acceso a material impreso

3.4.3 Acceso a biblioteca de recursos

3.4.4 Acceso secuencial a información

3.4.5 Realimentación
4.1.1 Grado de determinación de objetivos

4.1.2 Grado de selección de contenidos

4.1.3 Grado de opciones de navegación

4.1.4 Grado de selección de actividades

4.1.5 Grado de decisión

4.2.1 Trabajo orientado al dominio

4.2.2 Trabajo orientado al problema

4.2.3 Grado de orientación

Pragmático

4.3 Trabajo en pares, equipos y/o grupos

4.4 Soporte cognitivo

4.5 Soporte cognitivo

4.4.1 Intercambio vía foros
4.3.1 Relación de actividades individuales v/s actividades grupales

4.4.2 Intercambio vía correo electrónico

4.5.1 Asesoramiento de expertos

4.5.2 Uso de simulaciones

4.5.3 Aplicaciones reales 
Tabla 3

Definición de la métrica 1.1.1 Grado de determinación de objetivos

Título

Tipo

Sub-característica

Definición/comentarios

Tipo de criterio elemental

Escala de preferencia
1. Control centrado en el estudiante

\section{Atributo}

1.1.1 Grado de determinación de objetivos

Este atributo representa el grado de toma de decisión por parte del usuarioestudiante en la selección de los objetivos y la libertad que tiene para ello en su totalidad

Es un criterio de variable discreta, binario y absoluto, en donde se evalúa la posibilidad de seleccionar objetivos, siendo $0=$ no disponible; $1=$ disponible.

$\operatorname{CrE}\left(\mathrm{X}_{\mathrm{i}}\right)=\{(0,0),(1,100)$

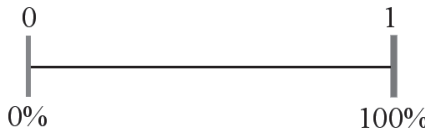

Tabla 4

Definición de la métrica 1.2.1 Número de materias

Título 2. Trabajo interdisciplinario

Tipo

Atributo

Sub-característica

1.2.1 Número de materias

Definición/comentarios

Este atributo representa el número de materias involucradas en los objetivos de aprendizaje y que se representan en las distintas actividades

Tipo de criterio elemental Es un criterio de variable discreta, multinivel y absoluto, en donde se evalúa la interdisciplinariedad, siendo $0=$ una materia; $1=$ dos materias y, $2=2$ o más materias

$\operatorname{CrE}\left(\mathrm{X}_{\mathrm{i}}\right)=\{(0,0),(1,60),(2,100)\}$

Escala de preferencia

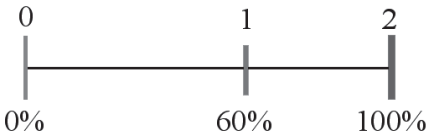

La métrica 1.2.1 de la tabla 2 ejemplifica este tipo de criterio en la tabla 4.

El criterio elemental multivariable discreta corresponde a un tipo de variable indirecta, en que su valor se determina por la conjugación de dos o más variables de tipo directas como en el caso de la métrica
1.1.5 de la tabla 2, que queda determinada por la métricas 1.1.1, 1.1.2, 1.1 .3 y 1.1.4, donde se asigna el valor de $25 \%$ a la presencia de cada una de ellas.

Un ejemplo de este tipo de criterio se ejemplifica en la tabla 5 , donde se establecen los resultados posibles de la 
Tabla 5

Definición de la métrica 1.1.5 Grado de decisión

Título

1. Control centrado en el estudiante

Tipo

Sub-característica

Definición/comentarios

Tipo de criterio elemental

Escala de preferencia
Atributo

1.1.5 Grado de decisión

Este atributo representa el grado de toma de decisión por parte del usuario-estudiante en su interacción con el sistema, basado en las subcaracterísticas 1.1.1 a 1.1.4

Es un criterio multivariable y discreto siendo, $\mathrm{D}_{1}=$ grado de determinación de objetivos; $\mathrm{D}_{2}=$ grado de selección de contenidos; $\mathrm{D}_{3}=$ grado de opciones de navegación y $\mathrm{D}_{4}=$ grado de selección de actividades $\mathrm{X}=\mathrm{F}\left(\mathrm{D}_{1}, \mathrm{D}_{2}, \mathrm{D}_{3}, \mathrm{D}_{4}\right), \mathrm{y}$ donde $\mathrm{X} \in\left\{\mathrm{X}_{1}, \mathrm{X}_{2}, \mathrm{X}_{3}, \ldots, \mathrm{X}_{16}\right\}$

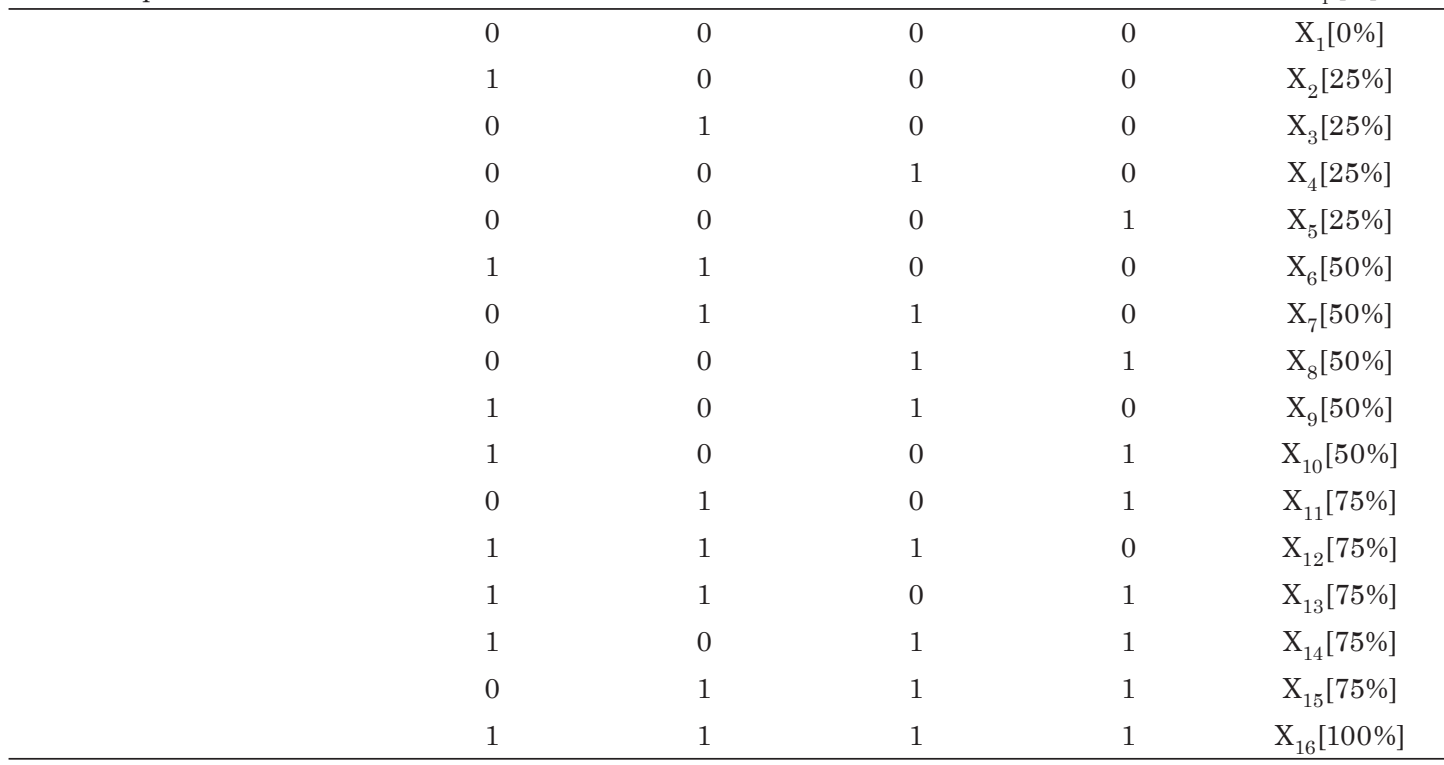

combinación de ellas. En dicha tabla se indica que a la presencia de una de las variables $\mathrm{D}_{\mathrm{i}}$ le corresponde un valor de calidad del $25 \%$, es decir, al tratarse de cuatro variables los valores posibles de calidad son: $0 \%$ si ninguna variable está presente, $25 \%$ si dos de ellas están presentes, $75 \%$ si tres variables se encuentran presentes y $100 \%$ si la totalidad de ellas están presentes.

\section{Conclusiones}

La propuesta presentada en este artículo, y que aún se encuentra en su fase de desarrollo, pretende una vez finalizado el trabajo establecer una metodología para la evaluación de calidad de los SHAE basados en Estilos de Aprendizaje en sus aspectos de interacción y actividades pedagógicas. 
Los aspectos aquí considerados corresponden sólo a la interacción pedagógica y basados en la teoría de estilos de aprendizaje planteado en (Alonso et al., 1997), y el detalle de las métricas correspondientes al estilo activo. Sin embargo, esta misma metodología es válida para los demás estilos de la misma teoría o de otra en que el SHAE se base.

Los atributos son determinados por las características del estilo, y mientras más precisas sean esas características más específicos serán los atributos. Esta misma inferencia es válida en la relación atributo-métricas, donde mientras más específicos y claros sean los atributos, mayores serán la especificación y objetivación de las métricas, permitiendo un adecuado y riguroso tratamiento matemático. Todo lo anterior asegurará una adecuada interpretación de los resultados de la calidad del sistema siempre bajo el prisma pedagógico.

\section{Agradecimientos}

Deseamos manifestar nuestro agradecimiento al grupo AWEG -Adaptive Web Engineering Group- de la Universidad de Salamanca por sus contribuciones e ideas en el desarrollo de este trabajo. Este trabajo ha sido parcialmente financiado por el proyecto ODISEAME (Open distance Interuniversity Synergies between Europe, Africa and Middle East), ref. EUMEDIS B7-41100/2000/2165-79 P546.

\section{Referencias bibliográficas}

Alonso, C., Gallego, D., Honey, P. Los Estilos de Aprendizaje. Procedimientos de diagnóstico y mejora. Bilbao: Ediciones Mensajero. 1997.
Bevan, N., Kirakowski, J. y Maissel, J. "What is Usability". Proceedings of the 4th International Conference on HCI, Stuttgart, Septiembre. 1991.

Bevan, N. "Quality in Use: Meeting User Needs for Quality". Journal of System and Software, Vol. 49, 89-96. 1999.

Leighton, H., García, F.J. Calidad en los Sitios Web Educativos. Technical Report (DPTOIA-IT-2003-002), Departamento deInformáticayAutomática, Universidad de Salamanca (España). http://tejo.usal. es/inftec/2003/DPTOIA-IT-2003-002. pdf. Noviembre. 2003.

Leighton, H. y García, F.J. "Atributos y métricas en sistemas hipermedia educativos adaptativos basados en el estilo de aprendizaje activo". En M. Moreno y F. García (Eds): Avances en gestión de proyectos y calidad del software. Departamento de Informática y Automática, Universidad de Salamanca. 25-34. 2004a.

Leighton, H., García, F.J. y López, R. "Determinación de atributos y métricas en sistemas hipermedia educativos adaptativos". En J. Sánchez, J. Gómez, M. Vega, B. Fernández y J. Bravo (Eds): Avances en informática educativa. VI Simposio Internacional de Informática Educativa, SIIE 2004. Universidad de Extremadura, Cáceres. España. 2004b.

Nielsen, J. Usability Engineering. New York: Academic Press Professional. 1993.

Olsina, L. Metodología Cuantitativa para la Evaluación y Comparación de la 
Calidad de Sitios Web. PhD. Thesis, Universidad Nacional de La PlataArgentina. 1999.

Olsina, L. "Métricas, Criterios y Estrategias para Evaluar Calidad Web". En Jornadas de Actualización en Informática de la Facultad de Ingeniería, JAIFI'02. En línea: http:// www.ing.unlpam.edu.ar/jaifi2002/ Jaifi2002.pdf. 2002 [última vez visitado: 20-january-2003].

Olsina, L., Bertoa, F., Lafuente, G., Martín, M., Katrib, M. y Vallecillo, A. "Un marco conceptual para la definición y explotación de métricas de calidad". En actas de Jornadas de Ingeniería de Software y Bases de Datos (JISBD’02), Madrid, España, Noviembre. 2002.

Olsina, L., Lafuente, G. y Rossi, G. "Specifying Quality Characteristic and Attributes for Web Sites". En S. Murugesan y Y. Deshpande (Eds): Web Engineering. Managing Diversity and Complexity of Web Application Development. Lecture
Notes in Computer Science. Springer Verlag LNCS 2016. 266-278. 2001.

Prieto, M., Gros, B., García, F.J. Modelos para la Elaboración de Materiales Hipermedia Adaptativos para el Apre ndizaje. Informe Técnico (DPTOIAIT-2003-003), Departamento de Infor mática y Automática, Universidad de Salamanca (España). http://tejo.usal. es/inftec/2003/DPTOIA-IT-2003-003. pdf. 2003.

Prieto, M., Leighton, H., \& García, F. "Adaptive educational hypermedia proposal based on learning styles and quality evaluation”. En P. de Bra \& W. Nejdl (Eds.), Adaptive Hypermedia and Adaptive Web-Based Systems, LNCS 3137 (pp. 316-319). Germany: Springer Verlag. 2004.

Reigeluth, C. M. y Moore, J. "Cognitive Education and the Cognitive Domain". En Reigeluth, C. M. (Eds.). A New Paradigm of Instructional Theory - Vol. II,. 51-68. USA: Lawrence Erlbaum Associates, Inc, Publishers (LEA). 1999. 
